\title{
Performance of Sentinel-2A Remote Sensing System for Urban Area Mapping in Malaysia via Pixel-Based and OBIA Methods
}

\author{
Adhwa Amir Tan ${ }^{1}$, Helmi Zulhaidi Mohd Shafri ${ }^{2, *}$ and Nur Shafira Nisa Shaharum ${ }^{2}$ \\ ${ }^{\text {I} M a l a y s i a n ~ S p a c e ~ A g e n c y ~(M Y S A), ~ J a l a n ~ T u r i, ~ K o m p l e k s ~ T e k n o l o g i ~ A n g k a s a, ~} 42700$ Banting, \\ Selangor, Malaysia \\ ${ }^{2}$ Department of Civil Engineering and Geospatial Information Science Research Centre (GISRC), \\ Faculty of Engineering, Universiti Putra Malaysia (UPM), 43400 Serdang, Selangor, Malaysia
}

('Corresponding author's e-mail: helmi@upm.edu.my)

Received: 22 June 2020, Revised: 24 March 2021, Accepted: 31 March 2021

\begin{abstract}
Sentinel-2A remote sensing satellite system was recently launched, providing free global remote sensing data similar to Landsat systems. Although the mission enables the acquisition of $10 \mathrm{~m}$ spatial resolution global data, the assessment of Sentinel-2A data performance for mapping in Malaysia is still limited. This study aimed to investigate and assess the capability of Sentinel-2A imagery in mapping urban areas in Malaysia by comparing its performance against the established Landsat- 8 data as well as the fusion datasets from combining Landsat-8 and Sentinel-2A datasets and using Wavelet transform (WT), Brovey transform (BT) and principal component analysis. Pixel-based and object-based image analysis (OBIA) classification approaches combined with support vector machine (SVM) and decision tree (DT) algorithms were utilized in this assessment, and the accuracy generated was analysed. The Sentinel-2A data provided superior urban mapping output over the use of Landsat- 8 alone, and the fusion datasets do not yield advantages for single-scene urban mapping. The highest overall accuracy (OA) for pixel-based classification of Sentinel-2A images is $84.77 \%$ by SVM, followed by $65.27 \%$ using DT. BT produced the highest OA for the fusion images of $78.40 \%$ with SVM and $52.21 \%$ with DT. For the object-based classification of Sentinel-2A images, the highest OA is $71.33 \%$ by SVM, followed by 76.38 $\%$ using DT. Similarly, the highest OA of fusion images is obtained by BT of $50.35 \%$ with SVM, followed by $65.66 \%$ with DT. From the analysis, the use of SVM pixel-based classification for medium spatial resolution Sentinel-2A data is effective for urban mapping in Malaysia and useful for future longterm mapping applications.
\end{abstract}

Keywords: Sentinel-2, Landsat-8, Urban mapping, Image classification, Object-based classification

\section{Introduction}

Changes in land use and land cover are important features for observing, studying, and assessing the urban environment. Urban growth is related to rapid population growth [1,2]. The urban landscape and environment significantly change over time, and the urban landscape is covered by different types of geographic objects in many spatial forms. Accurate information from land cover data is vital for enhancing urban planning and monitoring studies of landscape, ecosystem, risk assessment, environment and land data integration models [3]. Land cover information is also essential for modelling the dynamics of the urban environment and for the feasible improvement and effective resource and asset management [4]. The urban context is full of complexity because of the heterogeneous and highly fragmented environment [5]. Spatial and spectral information is essential for the recognition of urban objects from the remote sensing perspective. Identification and classification of the urban object are essential in gathering information and are used for future development. Such information plays a significant role in physical planning, producing up-to-date land statistics, and evaluating data on foliage and biomass [6].

The Sentinel-2A mission aims to provide extensive systematic coverage for land surface application for global-level usage. The system is equipped with a state-of-the-art payload design and offers 13 spectral bands with a high spatial resolution of $10 \mathrm{~m}$. Furthermore, Sentinel-2A has been designed for data continuity and enhancement of the Landsat and SPOT missions [7]. It offers incredible potential in focusing the satellite information aimed at the fine-scale mapping of settlement areas [8]. Value-added 
assessment of Sentinel-2A in distinguishing developed areas has shown potential in providing detailed urban land cover mapping. The system can produce a map of urban land cover and ecosystem, and its spatial characteristics can influence the research results [9].

Sentinel-2A data specifications are obtained from the Landsat-8 spectral resolution combined with the SPOT-5 spatial resolution [10]. Thus, the system has the same spatial resolution as SPOT-5 but in various spectral bands similar to Landsat-8. Subpixel landscape identification for the urban environment can be potentially carried out with the help of spectral separability analysis. However, separating some similar classes, such as mangroves and water bodies, is difficult in land-use monitoring [11]. Additional processes or information should be provided to distinguish the class and thus produce accurate land cover maps. This task can be accomplished with the fusion data.

Various algorithms and models have been developed to produce reliable land classification through remote sensing source and analysis with geographic information system (GIS). Most techniques have been applied to secure precise and dependable information from satellite images. The common classification approaches for remote sensing imagery can be categorized into (i) classifications based on pixels and (ii) classifications based on objects [12]. The pixel-based classification has become the most common approach for remote sensing imagery. This type uses multi-spectral information from the pixel imagery to assign the classes with similar spectral bands or others [13].

For example, a study was conducted on the classification mapping comparison using classifiers based on pixels, such as support vector machine (SVM), maximum likelihood (ML), and spectral angle mapper (SAM), for Landsat-8 and Radarsat-2 images [14]. The results indicated that the overall accuracy (OA) of SVM is higher than that of SAM and ML. Another similar study used 9 machine learning algorithms, namely, linear discriminant analysis, random forest (RF), k-nearest neighbor (kNN), SVM, multi-layered perceptron ensemble, multi-layered perceptron, boosting, ctree, and logarithmic regression for Sentinel-2A images [15]. The results indicated that RF obtains the best classification result, followed by $\mathrm{kNN}$ and SVM. On the other hand, the comparison on the classification accuracies was conducted between ML and SVM for Sentinel-2A and Landsat-8 images. The results showed that SVM produces a better result than ML [16].

In producing object-based image analysis (OBIA), researchers used shape, pattern, texture, and spectrum (spectral) indicators to describe the feature classes [12,17]. Min [29] generated object-based classification using multi-resolution image segmentation method combined with spectral and shape features. Segmentation results produced high visual homogeneous parcels for several different types of resolutions images with high recognition. Wang et al. [18] classified land covers for object- and pixelbased classification using SVM and RF classifiers. RF outperforms SVM in pixel-based analysis but obtains lower OA than SVM in object-based analysis. Gibril et al. [14] focused on feature extraction accuracy and showed that SVM achieves maximum accuracy for pixel-based classification and rule-based approach presents efficient overall classification accuracy for object-based classification. Object-based classification for Sentinel-2A images showed that Sentinel-2A obtains effective results in mapping urban land cover and ecosystem with SVM [19].

This study mainly aimed to investigate, assess, and compare the capability of Sentinel-2A with that of Landsat-8 for mapping urban land-use in Kuala Lumpur City, Malaysia, where the urban landscape comprises a complex, heterogeneous, and mixed land-use area. Comparing Sentinel-2A and Landsat-8 imagery has never been tested over Malaysia for urban applications. Therefore, this study is the first to investigate Sentinel-2A in Malaysia. It is hoped that the study results can help evaluate the data source and other application needs.

\section{Materials and methods}

\section{Study area and data}

The study area is the southern part of Kuala Lumpur between longitude $101^{\circ} 39^{\prime} 37.98^{\prime \prime}$ and $101^{\circ} 45^{\prime}$ 18.75" and latitude $3^{\circ} 7^{\prime} 8.39^{\prime \prime}$ and $3^{\circ} 4^{\prime} 26.78^{\prime \prime}$ (Figure 1). The area consists of different urban landscape characteristics and comprises residential area, commercial area, industrial area, airport, road, highway, railway, water bodies and vegetation-land use land; the total area coverage is approximately $25 \mathrm{~km}^{2}$. This area is still developing, and its demographics will change in the future with the Bandar Malaysia redevelopment project that supports the Greater Kuala Lumpur plan as a new engine of growth. 


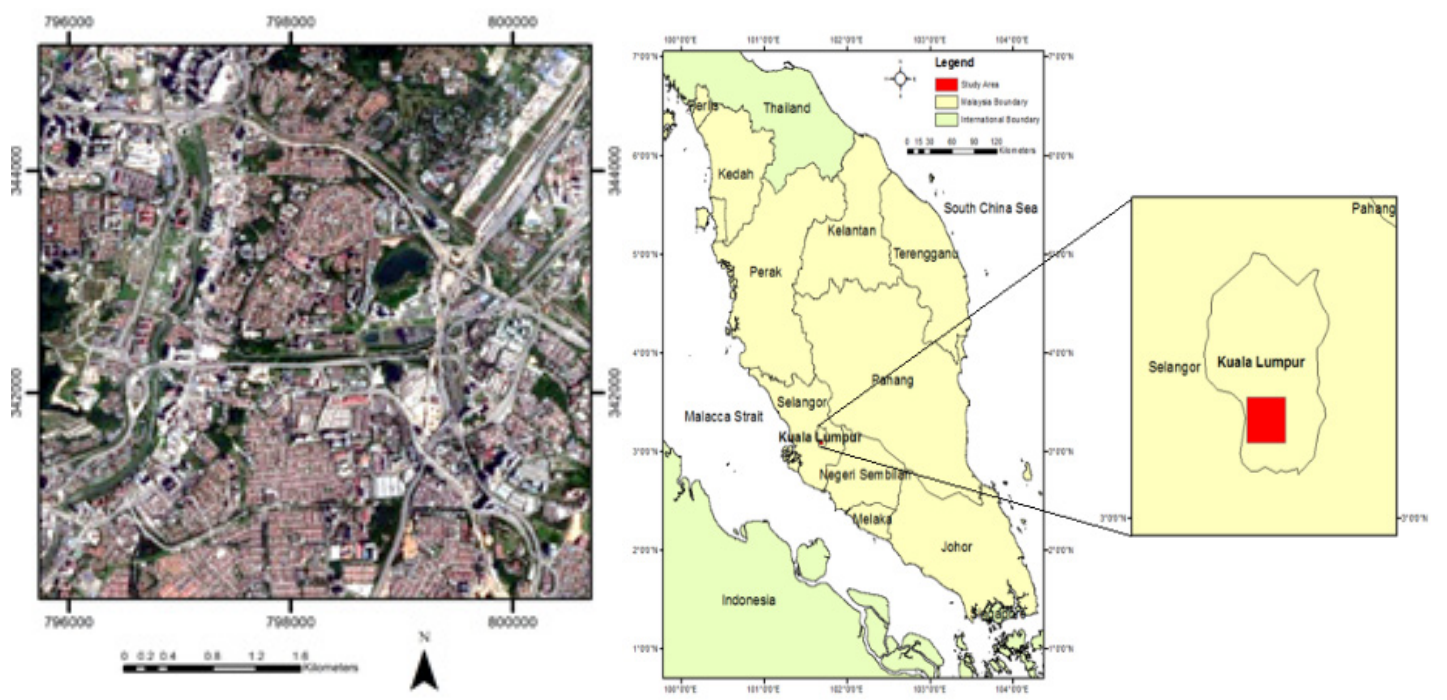

Figure 1 Google map image (left) and location of the study area.

Satellite images of Sentinel-2A and Landsat- 8 were acquired and processed. Sentinel-2A is the new satellite program under the European Commission (EC) alongside the European Space Agency (ESA) partnership and was launched on June 23, 2015. The satellite carries a multi-spectral camera and covers 13 spectral bands using spectral ranges from 443 to $2190 \mathrm{~nm}$ and $290 \mathrm{~km}$ swath width. The data product from Sentinel-2A has $10 \mathrm{~m}$ spatial resolutions of 4 visible and near-infrared bands, $20 \mathrm{~m}$ for 6 red-edge (shortwave infrared) bands, and $60 \mathrm{~m}$ of 3 atmospheric correction bands.

Over the years, the Landsat satellite program has provided the longest running acquisition of satellite imagery for earth resource monitoring among other satellite programs. Landsat-8, which was launched on February 11, 2013, is a joint project of NASA and USGS and obtains global medium resolution measurements through the visible, near-infrared, shortwave, and thermal infrared spectrum bands [16]. The satellite exhibits increased capabilities with a multi-spectral camera (Operational Land Imager, OLI) and the satellite covers 8 spectral bands $(430-2290 \mathrm{~nm})$ at $185 \mathrm{~km}$ swath width cross-track field of view. Landsat- 8 has $30 \mathrm{~m}$ spatial resolution of visible, near-infrared, and shortwave bands, and 15 $\mathrm{m}$ of a panchromatic band.

In this study, Sentinel-2A images were downloaded from the Copernicus Open Access Hub from the ESA. The images were acquired on March 30, 2016 and radiometrically pre-processed by the data provider. The multispectral Landsat- 8 images were acquired on March 30, 2016. The images were obtained through the US Geological Survey (USGS), Earth Resources Observation and Science Centre, and radiometrically pre-processed by USGS. Although both satellites have similar spectral coverage (430 - $2300 \mathrm{~nm}$ ), the levels of improvement between them still need to be studied. The selected spectral bands and spatial resolutions are categorized in Table 1. For the classes of land use and land cover, the total categories have been divided into 11 classes (Table 2).

Table 1 Selection Sentinel-2A and Landsat-8 spectral and spatial resolution.

\begin{tabular}{lclc}
\hline \multicolumn{1}{c}{ Sentinel-2A bands (nm) } & Resolution (m) & \multicolumn{1}{c}{ Landsat-8 bands (nm) } & Resolution (m) \\
\hline Band 2 (458 - 523) & 10 & Band 2 $(450-510)$ & 30 \\
Band 3 (543 - 0.578) & 10 & Band 3 $(530-590)$ & 30 \\
Band 4 (650 - 680) & 10 & Band 4 $(640-670)$ & 30 \\
Band 5 (698 - 713) & 20 & Band 5 $(850-880)$ & 30 \\
Band 6 (733 - 748) & 20 & Band 6 $(1570-1650)$ & 30 \\
Band 7 (765 - 785) & 20 & Band 7 $(2110-2290)$ & 30 \\
Band 8 (785 - 900) & 10 & Band 8 $(500-680)$ & 15 \\
Band 8a (855 - 875) & 20 & & \\
Band 11 (1565 - 1655) & 20 & & \\
\hline
\end{tabular}


Table 2 List of classes for the classification process.

\begin{tabular}{ccc}
\hline No. & Class & Definition \\
\hline 1 & Water & Surface of water bodies, such as lake and river \\
2 & Grass & Vegetation comprising short plants \\
3 & Tree & Vegetation comprising structured high plants \\
4 & Soil & Bare surface of earth and cleared area \\
5 & Commercial & Commercial area of business complexes, shopping malls, and shops \\
6 & Road & Access way comprising normal road and highway \\
7 & Pavement & Hard surface of a road or sidewalks \\
8 & Shadow & Dark surface coming from building or cloud due to sunlight angle \\
9 & Settlement 1 & Built areas that have been developed or clustered building (residential, \\
10 & Settlement 2 & transportation, station, and airport) \\
11 & Settlement 3 & \\
\hline
\end{tabular}

Methods

The methodology for the research started with pre-processing of Sentinel-2A and Landsat-8 images. Then, data fusion process and image classification were conducted. Finally, the accuracy generated was assessed. Figure 2 shows the data processing workflow in detail.

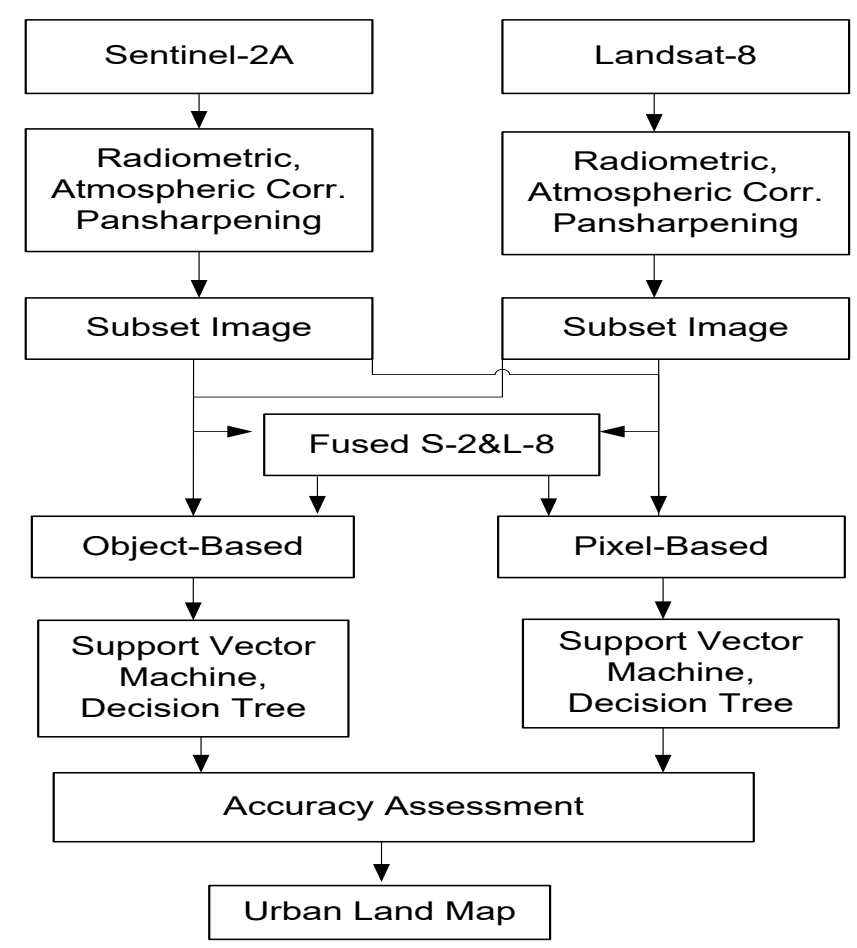

Figure 2 Workflow for Sentinel-2A and Landsat-8 data processing.

\section{Digital pre-processing}

Sentinel-2A data were converted to surface reflectance (Bottom of Atmosphere, BOA) using a SNAP tool function called Sen2Cor, which allows atmospheric corrections to the Level-1C product (Top of Atmosphere, TOA). Sen2Cor has 2 main parts: (i) scene classification, which provides a classification map of cloud, shadow, water, snow, vegetation and soil/desert classes; and (ii) atmospheric correction, 
which generates BOA reflectance from TOA reflectance [21]. Resampling was conducted because some of the spectral bands on Sentinel-2A present a native spatial resolution of $20 \mathrm{~m}$. The $20 \mathrm{~m}$ resolution bands were increased by means of cubic interpolation. Most high-resolution $(10 \mathrm{~m})$ spectral bands were used as panchromatic bands for low spatial resolution $(20 \mathrm{~m})$ bands in the pan-sharpening process. This process was applied to Bands 5, 6 and 7 with the average Bands 4 and 8. For Band 8a, B and 8, they were directly utilized as the panchromatic channel [21]. Full resolution of Sentinel-2A bands was processed using Gram-Schmidt pan-sharpening.

Atmospheric correction was established for Landsat-8 images by using the FLAASH function for multi-spectral images. The digital number was converted to radiance and reflectance to generate surface reflectance to the multi-spectral images. Thereafter, the output with a panchromatic channel of $15 \mathrm{~m}$ was pan sharpened using Gram-Schmidt pan-sharpening to improve spatial resolution. For the pan-sharpened output images of Sentinel-2A and Landsat-8, the band math function was adopted using Eq. (1) such that all the image values synchronize to the reflectance values. According to Singh [22], a mathematical operation that performs band by band should be used to allow correcting the negative values and replacing them with values between 0 and 1 .

$(B 1 L E 0) * 0+(B 1 G E 10000) * 1+(B 1$ GT 0 and B1 LT 10000) $*$ float $(B 1) / 10000$

where B1 is a channel band, LE is denoted as less than or equal, GE is denoted as greater than or equal, GT is denoted as greater than and LT is denoted as less than.

\section{Data fusion}

Image fusion is a process of combining various images from diverse spectral bands, spatial resolutions, and radiometers to acquire high-quality results. In this process, 3 combinations of fusion methods, namely, wavelet transform (WT), Brovey transform (BT), and principal component analysis (PCA), were accomplished for Sentinel-2A and Landsat-8. The fusion process was conducted using ENVI and ERDAS Imagine image processing software. Pre-processing is important to allow alignment of all the images with one another and thus eases the construction of pan-sharpened images.

PCA methods convert the input channel into a set of uncorrelated orthogonal components and use the eigenvectors from the covariance matrix [23]. This method replaces the first principal component in the image with the histogram-matched PAN image. The output image will contain the new spatial information while the spectral information remains in other principal components. BT is based on the normalization of the spectral modelling from the input bands over addition, subtraction and arithmetic ratio. BT preserves the relative spectral contribution of each pixel in the fusion process. The multispectral band input is normalized and multiplied with the high spatial resolution image before the output image is generated [24]. The wavelet method maintains the spectral qualities from the image unlike other approaches, such as PCA and HIS [18]. WT reconstructs multi-scale signals of the spectral characteristic to describe object features from the original image.

\section{Image classification}

Classification approaches can be divided into 2 types, namely, pixel-based and object-based classification. The training and sample areas for each object feature need to be created to perform the classification and assess the accuracy [25]. The 2 methods have been applied to existing Sentinel-2A and Landsat- 8 images and fusion images. These approaches use 2 types of classifiers, namely, SVM and DT, to produce urban classification maps. The suitable classifier can be selected by testing which classifier can produce a classification result of high accuracy.

SVM is a non-parametric statistical learning algorithm under the supervised classification category and can achieve excellent classification accuracy [3]. The classifier divides the classes by use of an optimal hyperplane that makes the most of the margins of multi-dimensional feature space. Figure 3 illustrates the SVM concept in creating the classes. 


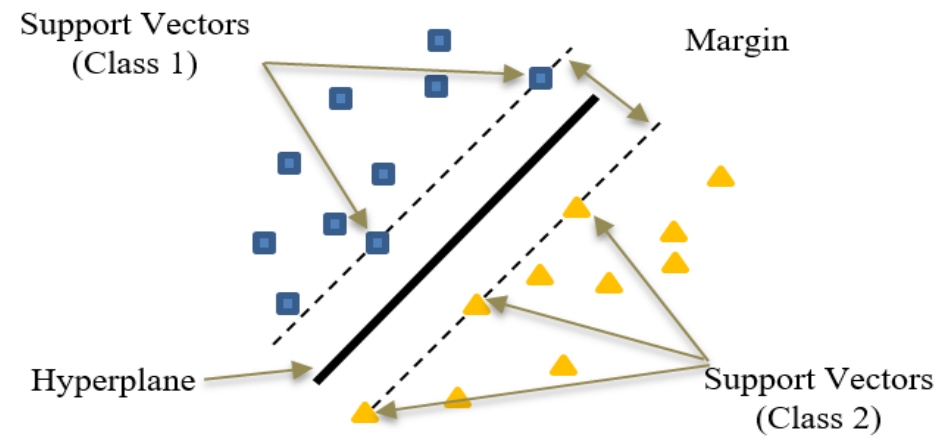

Figure 3 SVM concept in dividing classes.

The data near the hyperplane are known as support vectors and can be used as the training set for classification. In this study, the radial basis function (RBF) was selected. Eq. (2) shows the RBF of SVM for pixel-based classification in ENVI. The kernel parameter is 0.3333 for $g$ (gamma term for kernel function) and penalty parameter is 100 and 0 for pyramid level and threshold. For the object-based classification in eCognition Developer, the best kernel is Linear and C parameter is 2.

$K\left[x_{i}, x_{j}\right]=\exp \exp \left[-g\left\|x_{i}-x_{j}\right\|\right]^{2}, g>0$

DT is a knowledge-based algorithm with the input of partitioning provided by the user. The "divide and conquer" concept separates the input at the root node and reprocesses the input again at the interior nodes until final classes are created at the terminal nodes. The root and interior nodes can be used to select the input parameters. The classification procedure is applied through a rule set that determines the path from the root node to the terminal node [26]. The final node is the classed object. For the pixel-based classification in the current study, 5 DT models were created on the basis of the interpretation analysis from the spectral response of each band. Figure 4 shows the example of DT for Sentinel-2A. Meanwhile, the input of DT for object-based classification was obtained from the selected training samples.

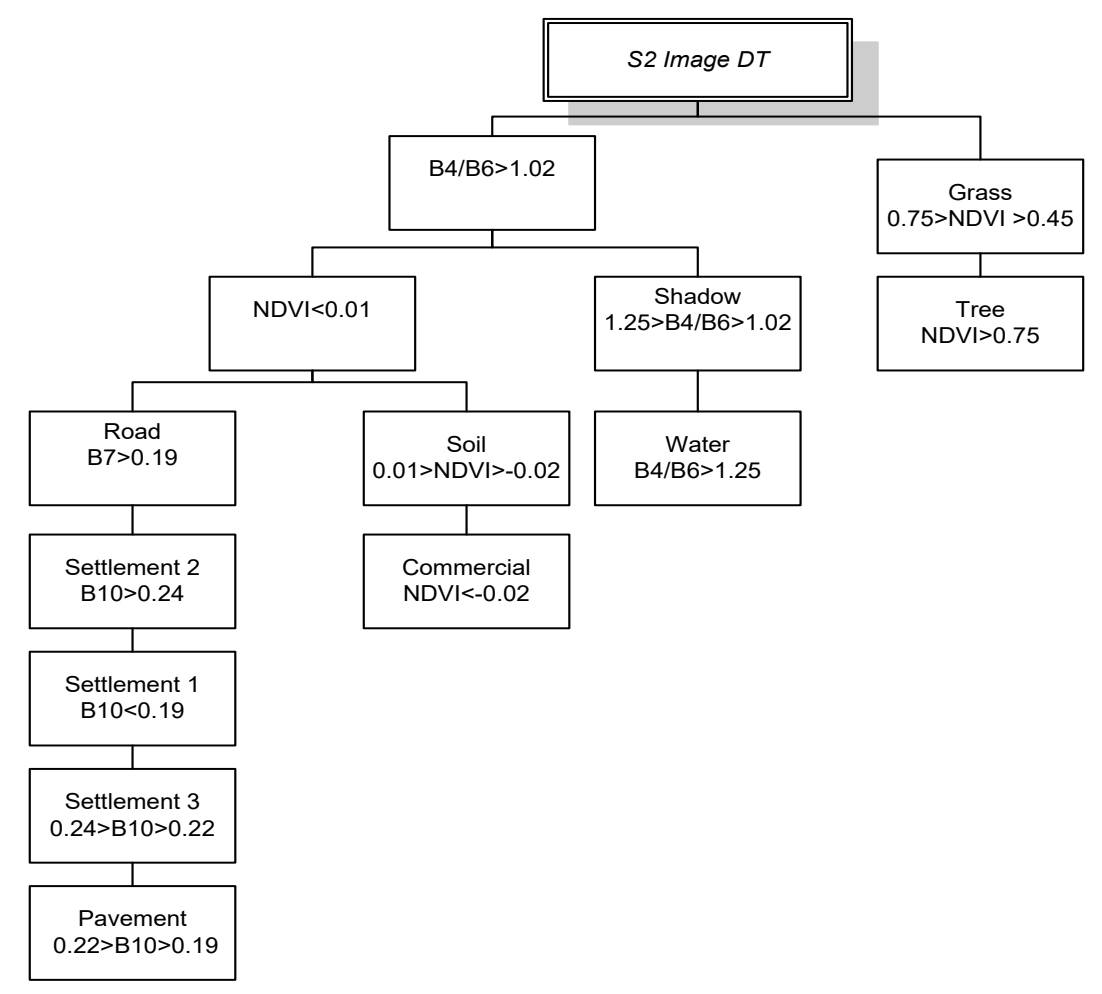

Figure 4 DT classification structure of Sentinel-2A image. 


\section{Data analysis}

The fusion image result was analysed, and the accuracy was assessed. Statistical analysis was conducted to analyse the changes in the image property parameters before and after the fusion process. The fusion images were evaluated on the basis of mean value, standard deviation, root-mean-square error, signal-to-noise ratio, and the normalization value from the spectral response. The comparative analysis is done to assess the changes in the reflectance value before and after the fusion process.

The accuracy of the classification result was evaluated on the basis of adding the number of the correctly classified areas and dividing it with the total number of reference areas for each feature class. The reference area was selected using the random sampling method and was created by point, line and polygon. The total number of pixels used in the research is 2,453 , where $48 \%(1,166)$ of the total pixels for training datasets while $52 \%(1,287)$ for testing datasets. The area was validated by the use of Google Map and Street Map application. The classification output and the independent observation were also statistically assessed for the probability error measurement [26]. The error matrix of feature classes from the classification result should be calculated for the accuracy result subject to OA, kappa coefficient (KC), and user and producer accuracies. This validation process was conducted to confirm the reliability and acceptability of the classification result. The accuracy assessment was performed on all the classified maps.

\section{Results and discussion}

The classification result was evaluated in terms of $\mathrm{OA}$ and $\mathrm{KC}$. The classes were selected using visual interpretation, spectral values and knowledge on the research area. The classification process was performed using the same sample areas and the training area to achieve constant variables.

\section{Pixel-based classification}

All pixel-based classification was conducted using SVM and DT classifiers. The results of SVM classification are shown in Figure 5. The OA and $\mathrm{KC}$ have indicated that S2SVM classification map result has a high percentage with strong agreement between classification and truth values. Visually, SVM pixel-based classification shows that L8SVM produces more generic feature class groups than the other methods. This result is expected because of the low resolution of L8. Therefore, L8 has limited capabilities to distinguish all the feature objects and create general and mixed classes on the basis of the spectral value. The fusion images using PCA, BT and WT methods have been unable to improve the feature extraction or interpretation compared with the single Sentinel-2A image in SVM classifier.

With the increased spatial resolution of the fusion images, fine classification still cannot be produced. The results for pixel-based classification show that all methods based on SVM classifiers produce higher OA than those based on DT classifiers. S2SVM $(84.77 \%)$ obtains the highest OA, followed by L8SVM (79.02 \%), BTSVM (78.40\%), PCASVM (78.01\%), and WTSVM (57.58 \%).

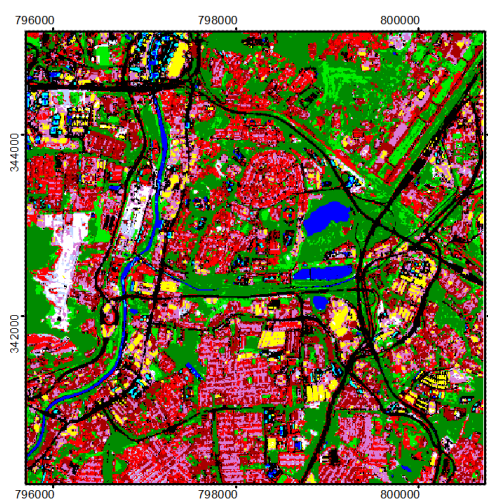

(a)

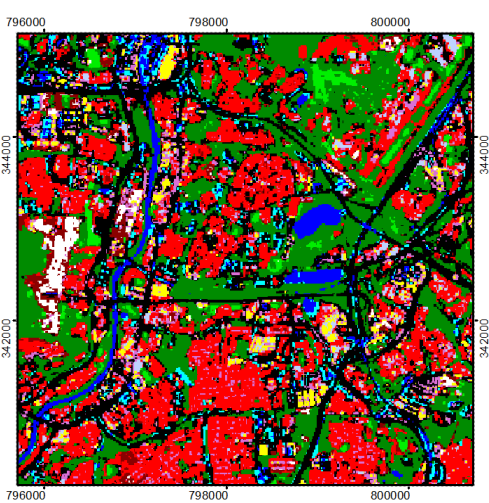

(b) 


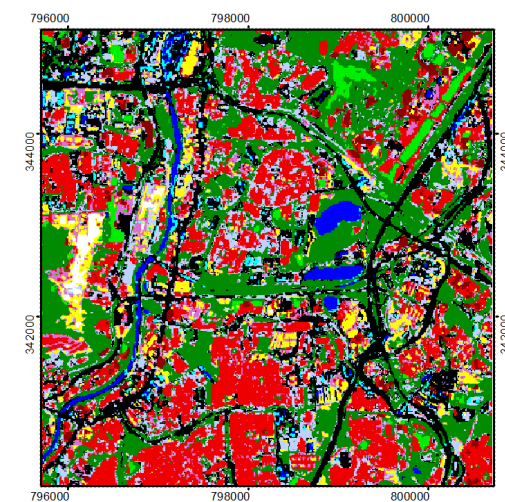

(c)

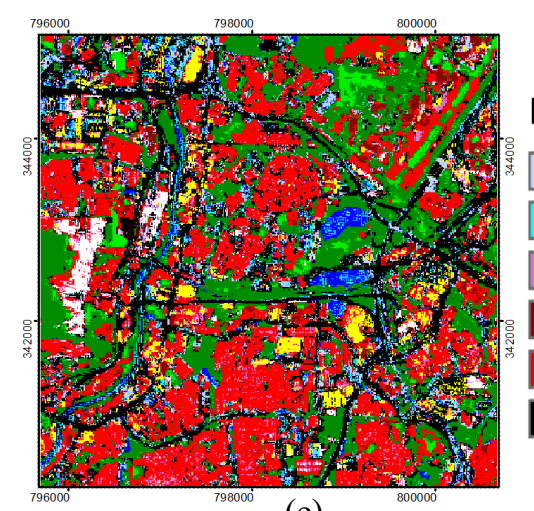

(e)

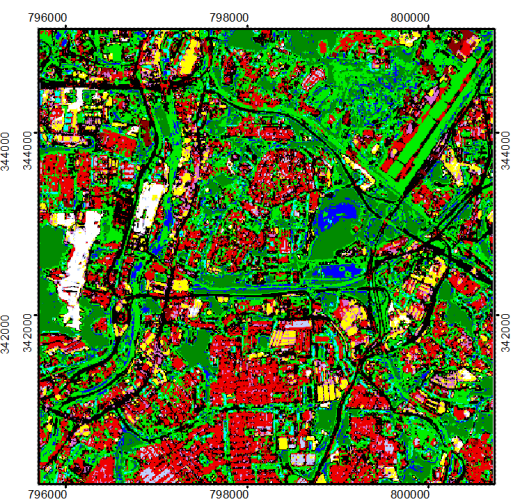

(d)

\section{Legend}
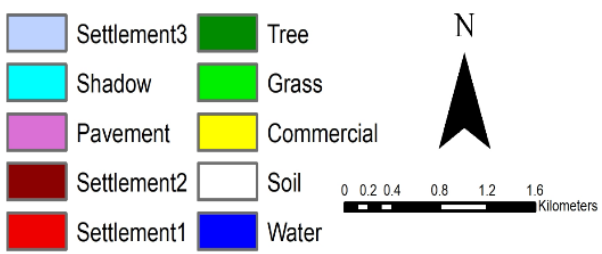

Road

Figure 5 SVM pixel-based classification: (a) Sentinel-2A, (b) Landsat-8, (c) PCA, (d) BT and (e) WT.

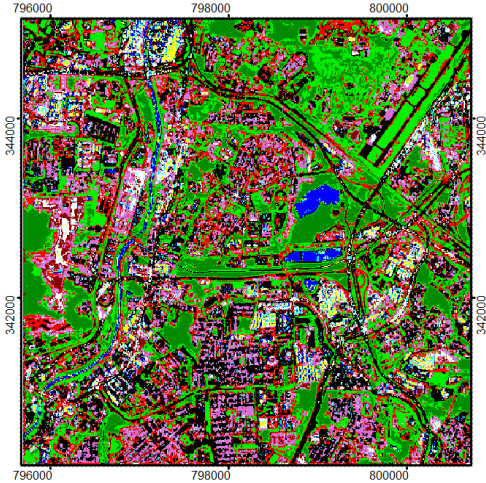

(a)

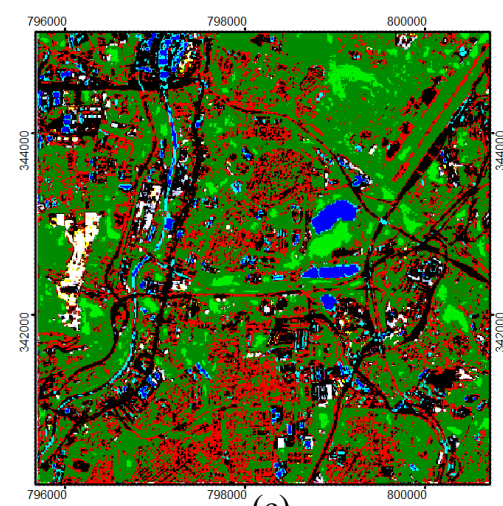

(c)

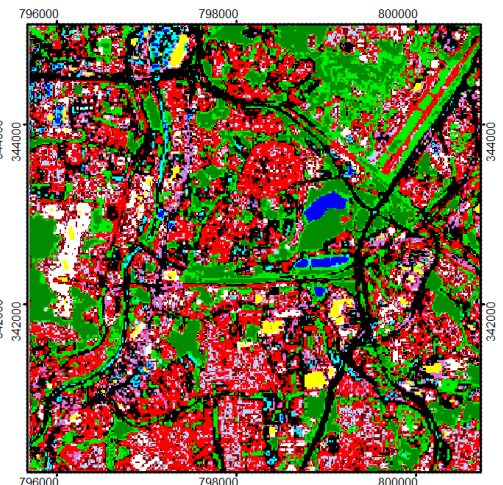

(b)

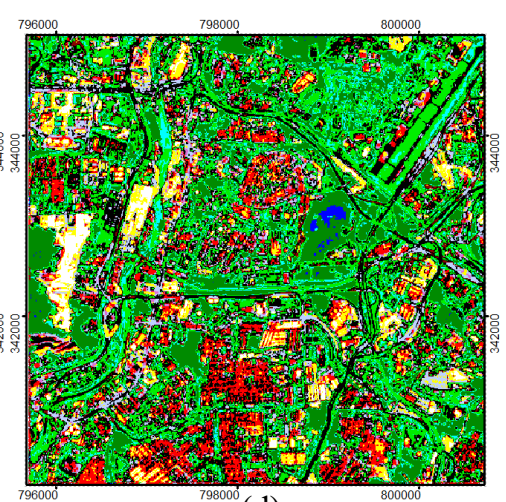

(d) 


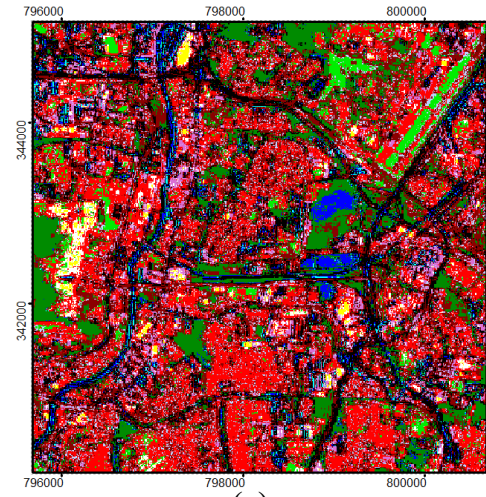

(e)

\section{Legend}

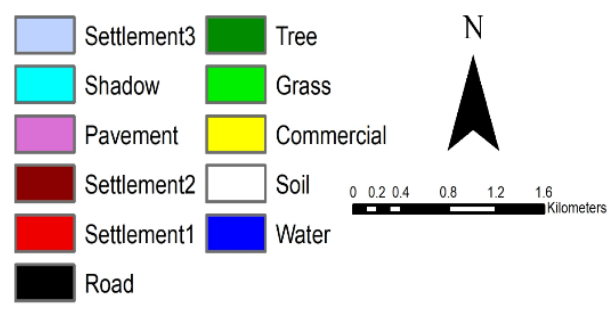

Figure 6 DT pixel-based classification: (a) Sentinel-2A, (b) Landsat-8, (c) PCA, (d) BT and (e) WT.

The results of DT classification are presented in Figure 6. Visually, S2DT and L8DT generate better feature classes than the fusion images (PCADT, BTDT, and WTDT). Some of the feature classes are undetected in the fusion images. In the case of PCADT, the settlement area is barely detected. BTDT detects few water bodies features and WTDT produces generic feature classes. For the methods based on DT classifier, most of the fusion image result is below $60 \%$. S2DT $(65.27 \%)$ obtains the best classification performance, followed by L8DT $(62.55 \%)$ and BTDT $(52.21 \%)$. The low performing pixel-based classification methods are PCADT (45.61\%) and WTDT (45.61\%). All the OA and KC results of pixel-based classification methods are presented in Figure 7.

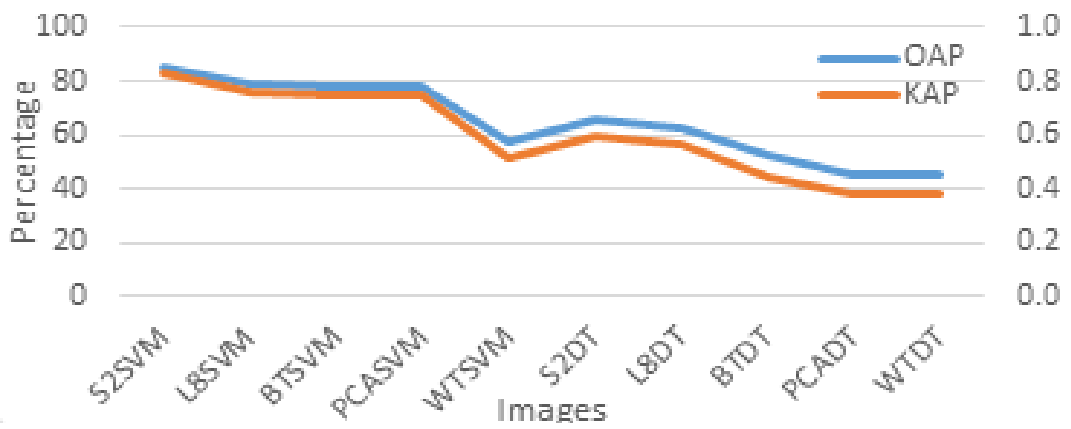

Figure $7 \mathrm{OA}$ and $\mathrm{KC}$ results for pixel-based.

$\mathrm{KC}$ was calculated using the sample and training areas to measure the association degree among the categorical variables (i.e., the feature classes). $\mathrm{KC}$ was used to measure the association degree between the classification map and data reference. This agreement can be obtained based on the error matrix of the feature class. Jensen [28] mentioned that a value of $\mathrm{KC}$ greater than 0.80 means a strong agreement. On the contrary, a value of $\mathrm{KC}$ lesser than 0.40 means a poor agreement. Meanwhile, a value of $\mathrm{KC}$ between 0.40 and 0.80 means a moderate agreement. As shown in Figure 7, the ranking of $\mathrm{KC}$ is similar to OA. The KC of DT with nearly $8 \%$ is much higher than SVM with only $2 \%$. This finding indicates that all SVM pixel-based classification images produce stronger agreement than all DT pixel-based classification images. Therefore, SVM classifier is much better with the sample target distribution in pixel-based classification.

\section{Object-based classification}

All object-based classification was also conducted using SVM and DT classifiers. The image analysis was achieved with the e-Cognition software to generate the shape and texture of the feature objects. Multi-resolution segmentation was implemented to the images to separate and shape the objects (Table 3). 
Table 3 Object-based parameters.

\begin{tabular}{lccc}
\hline Image & Scale parameter & Shape & Compactness \\
\hline L8 & 0.7 & 0.5 & 0.5 \\
S2 & 0.5 & 0.5 & 0.5 \\
L8S2 BT & 0.7 & 0.5 & 0.5 \\
L8S2 PCA & 0.7 & 0.1 & 0.5 \\
L8S2 WT & 0.8 & 0.5 & 0.5 \\
\hline
\end{tabular}

The segmentation results show that the object's shape is not constructing some of the following features because all the borders are square. Mixed classes still appear in object-based classification due to the spectral response layer information, although the shape and texture are added to the classification process. Visually, only S2 produces a significant result compared with the other methods based on SVM classifier. None of the fusion images generate a good result in feature extraction of 11 classes although the training sample is correctly selected. Figure 8 shows the results of SVM classification. S2SVM $(71.33 \%)$ produces the highest OA, followed by L8SVM (54.39\%), BTSVM (50.35\%), WTSVM (45.84 $\%)$ and PCASVM (41.34\%).

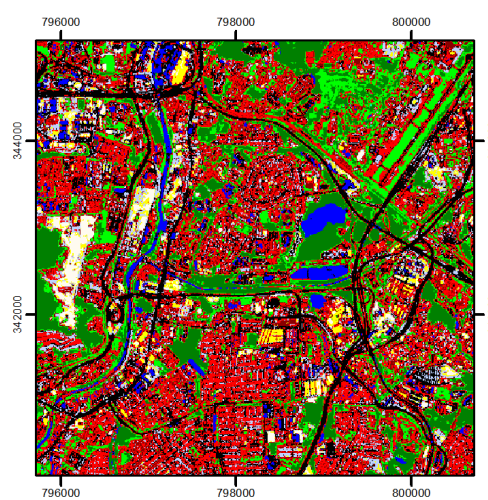

(a)

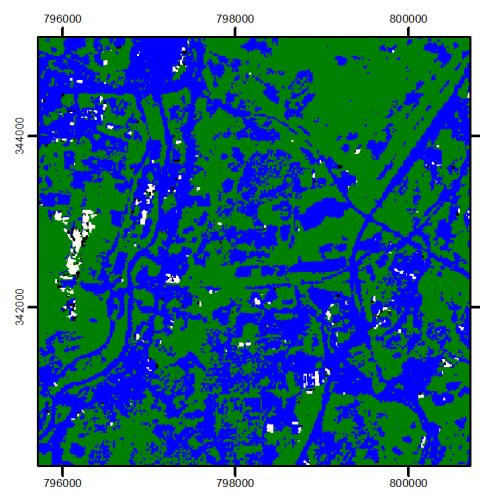

(c)

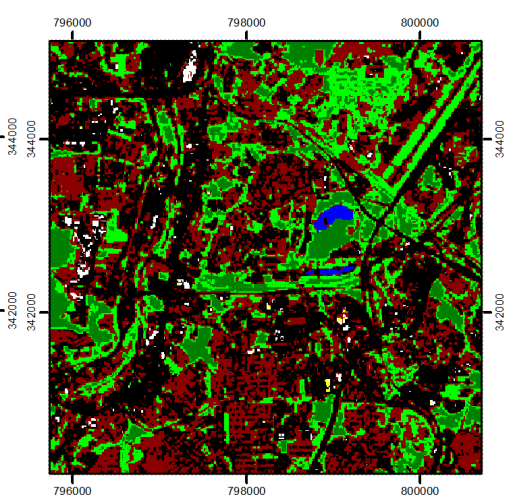

(b)

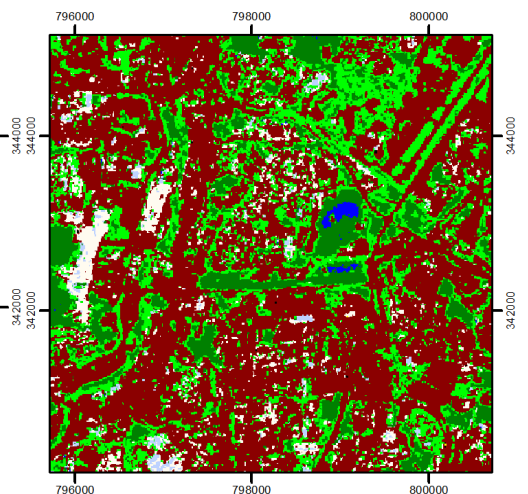

(d) 


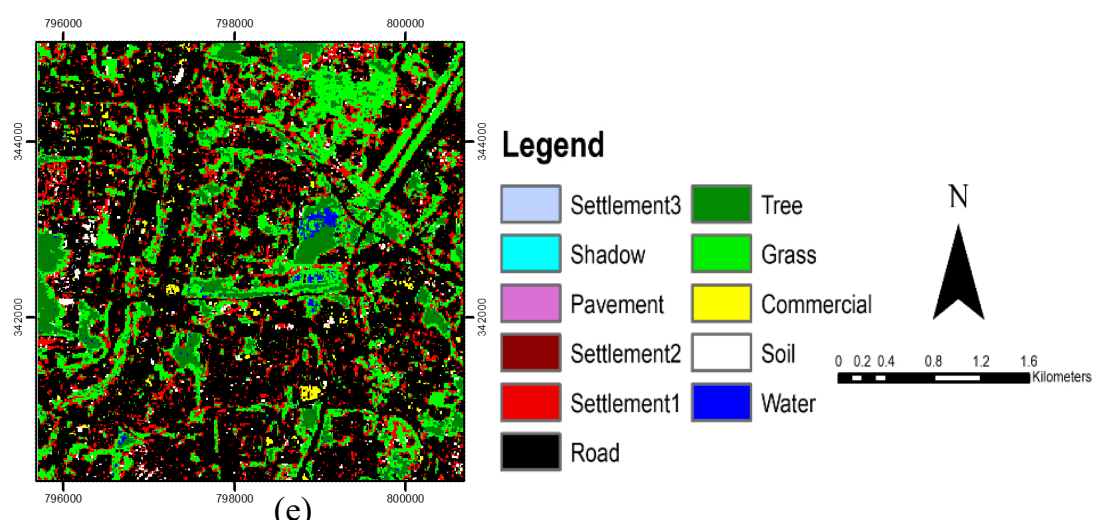

Figure 8 SVM object-based classification: (a) Sentinel-2A, (b) Landsat-8, (c) PCA, (d) BT and (e) WT.

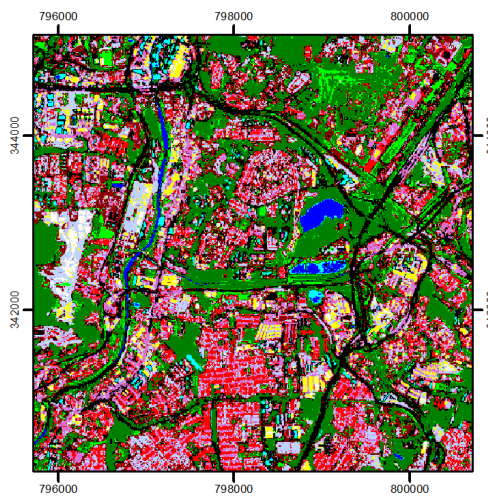

(a)

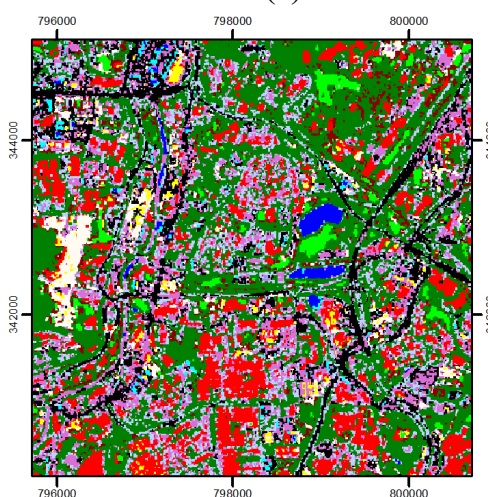

(c)

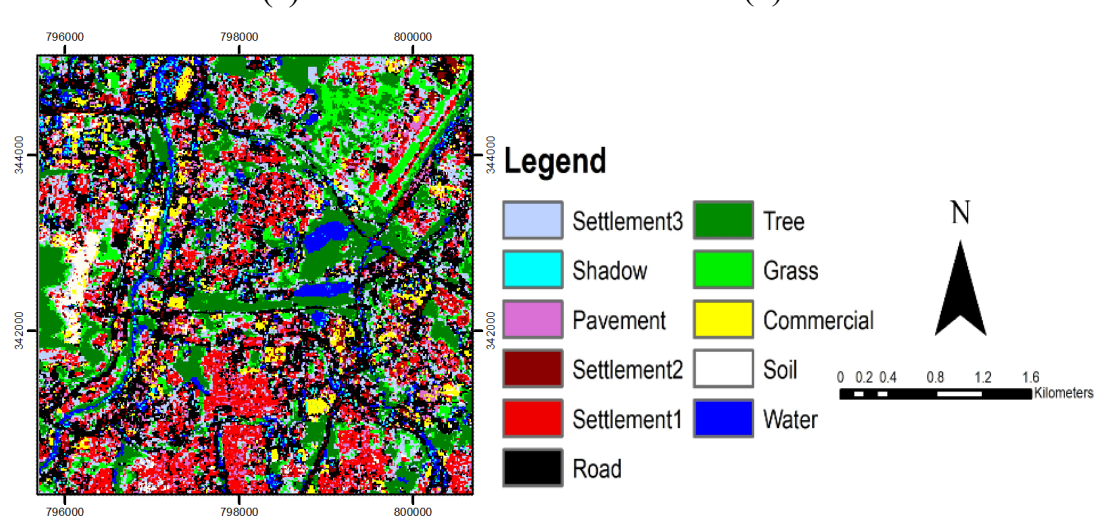

(e)

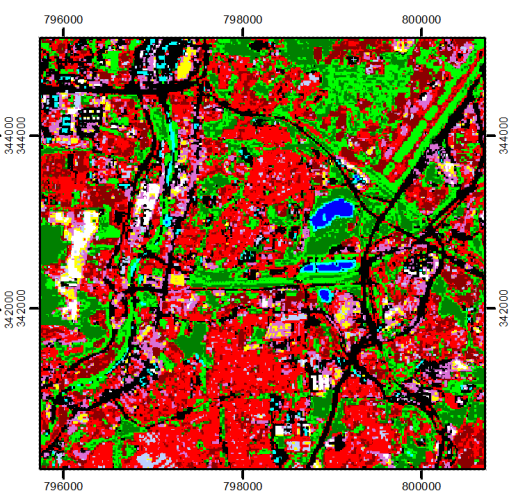

(b)

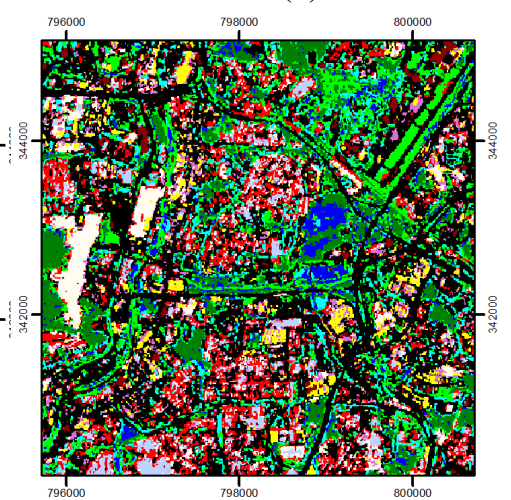

(d)

Figure 9 DT object-based classification: (a) Sentinel-2A, (b) Landsat-8, (c) PCA, (d) BT and (e) WT. 
The results of DT object-based classification are shown in Figure 9. All images produce the 11 classes. S2DT and L8DT generate better feature classes than other methods. Surprisingly, L8SVM obtains a better classification map than the fusion images. However, S2DT obtains finer feature shapes than L8SVM. For PCADT, the grass feature is simple and is taken over by the tree features. BTDT transforms tree features to water features. The road feature is extended and covers the settlement feature. Meanwhile, the grass feature replaces the water feature in the river area. In WTDT, the water feature is generated in the settlement and tree areas. A large number of mixed classes are found among the commercial and soil features.

Among the DT classification methods, S2DT (76.38 \%) obtains the highest OA, followed by L8DT (74.98 \%), BTDT (65.66 \%), WTDT (61.93\%) and PCADT (57.11\%). The OA and KC results of objectbased classification methods based on SVM and DT classifiers are presented in Figure 10. The objectbased classification results show a contrast effect unlike the pixel-based classification results. Therefore, SVM classifier generates lower OA than DT classifier.

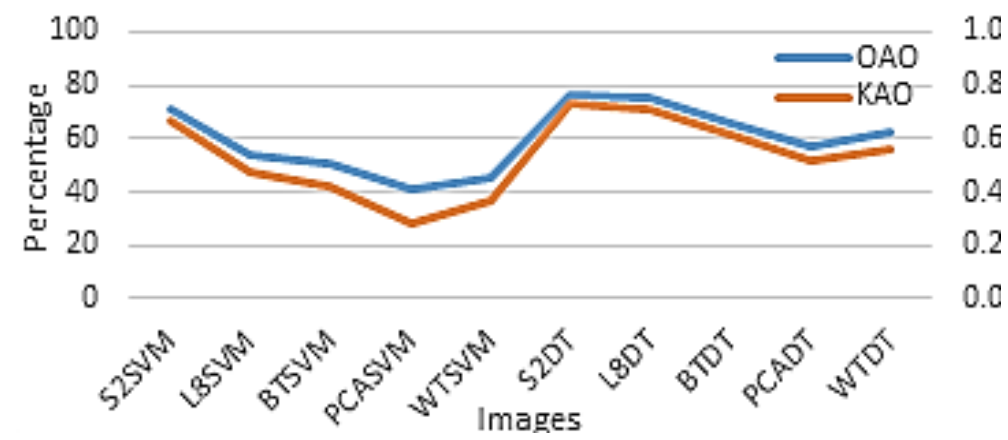

Figure $10 \mathrm{OA}$ and $\mathrm{KC}$ results for object-based classification.

Similar to the $\mathrm{KC}$ of pixel-based classification methods, that of the object-based classification methods was ranked based on the OA position. In this case, the $\mathrm{KC}$ of SVM with $13 \%$ is much higher than that of DT with $4 \%$. Therefore, DT classifier obtains a stronger agreement in the object-based classification than SVM classifier. In other words, the distribution sample targets in object-based classification are improved by DT classifier unlike SVM classifier. Figure 11 shows the OA comparison of pixel-based (OAP) and object-based $(\mathrm{OAO})$ classification results. $\mathrm{OA}$ and $\mathrm{KC}$ with different image types and classification approaches are shown in Table 4.

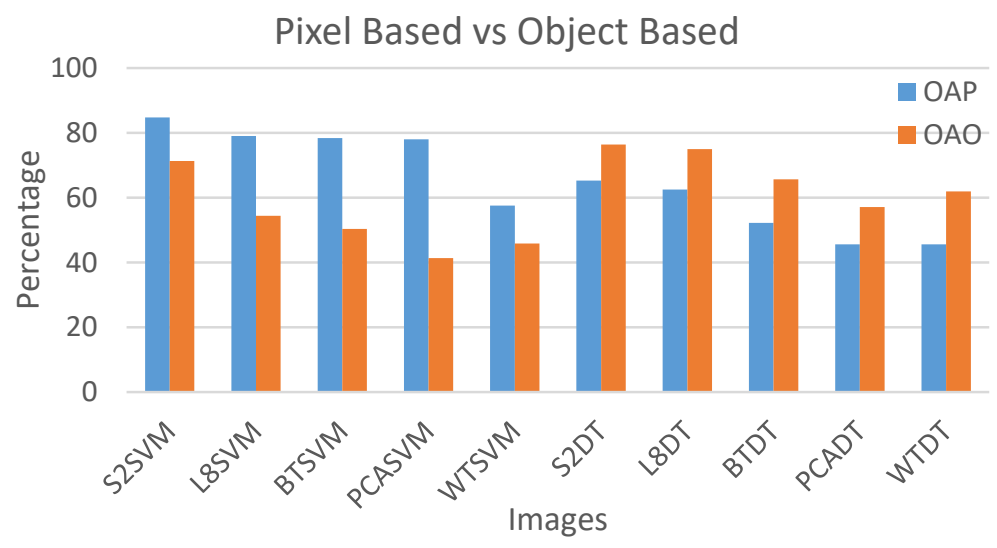

Figure 11 Comparison of pixel-based (OAP) and object-based (OAO) classification results. 
Table $4 \mathrm{OA}$ and $\mathrm{KC}$ with different image types and classification approaches.

\begin{tabular}{lcccccc}
\hline \multirow{2}{*}{\multicolumn{2}{c}{ Image type }} & \multirow{2}{*}{$\begin{array}{c}\text { Classification } \\
\text { approach }\end{array}$} & \multicolumn{2}{c}{ Pixel-based } & \multicolumn{2}{c}{ Object-based } \\
\cline { 3 - 6 } & & OA (\%) & Kappa & OA (\%) & Kappa \\
\hline Optical Image & Sentinel-2A & SVM & 84.77 & 0.83 & 71.33 & 0.67 \\
& & DT & 65.27 & 0.60 & 76.38 & 0.73 \\
& Landsat-8 & SVM & 79.02 & 0.76 & 54.39 & 0.47 \\
& & DT & 62.55 & 0.56 & 74.98 & 0.71 \\
Fusion Images & BT & SVM & 78.40 & 0.75 & 50.35 & 0.42 \\
& \multirow{4}{*}{ PCA } & DT & 52.21 & 0.45 & 65.66 & 0.61 \\
& & SVM & 78.01 & 0.75 & 41.34 & 0.28 \\
& \multirow{2}{*}{ WT } & DT & 45.61 & 0.38 & 57.11 & 0.52 \\
& & SVM & 57.58 & 0.51 & 45.84 & 0.37 \\
& & DT & 45.61 & 0.38 & 61.93 & 0.56 \\
\hline
\end{tabular}

The graph patterns in Figure 11 show that the SVM classifier produces good classification results in pixel-based classification. Meanwhile, the DT classifier strongly evaluates the feature classes in objectbased classification. Based on all the classification results, the feature classes can be justified. All the classification processes and accuracy assessments used the same location of training and sample areas. In Table 4, some of the $\mathrm{OA}$ and $\mathrm{KC}$ values are too different from each other. There are 2 possibilities that this may happen. First, it is about the sampling technique, and second, the interpretation of OA and KC on the data.

For the sampling technique, utilizing stratified random sampling may improve the value of OA and $\mathrm{KC}$ because this method enables the description of the entire population with a smaller number of samples. A large difference between $\mathrm{OA}$ and $\mathrm{KC}$ may be caused by some of the feature classes having a large majority and being well described compared to other classes. OA is, therefore an optimistic index that shall provide high value but less agreement between 2 datasets (samples).

On the feature classes' observation, the water and shadow features are difficult to separate and are mixed in some areas. Both features exhibit low spectral response from the spectral curve and visual interpretation because of their nearly black and dark blue color. Thus, the water feature is detected besides the shadow or water feature only. Several swimming pools are located in the study area. However, this part has not been selected as water class and does not appear in the classification result. The area Desa Cindaimas is classified as water and shadow. The area is agricultural (garden plant) that is covered by sun shade netting (black material).

Soil, commercial, and settlement are also mixed, as they present the same features because of their similar spectral response. The settlement feature is categorized into 3: settlement1, settlement 2 and settlement3. The reason is that the buildings are made of different types of surface material as obtained by the spectral response and visual interpretation. Some objects in the study area belong to 2 feature classes: Tall buildings of roof and wall reflecting different spectral responses.

Buildings and infrastructures (sewerage plants) are included in the road class because of their similar spectral response. Some roads reflect the pavement and settlement response. This case is true in the airport runway and the toll area because the build-up material and road surface can be a concretebased source. Grass and tree classes are straightforwardly determined in general because their spectral response differs from the others. Grass and water are usually mixed. This case is true in the Klang River and Sungai Kerayong channel. The reason is due to the water quality and the water flow condition at the river and channel. Grass and trees are also misclassified.

\section{Conclusions}

This research is the first to analyse the potential of the Sentinel-2A remote sensing system for mapping urban areas in Malaysia. The results show that Sentinel-2A data provide superior urban mapping output over the use of Landsat- 8 alone and that the fusion datasets do not yield advantages for singlescene urban mapping. In other words, fusion images only introduce complexities in analysing images. The Sentinel-2A imagery alone can provide fine and detailed results. 
For medium-resolution satellite imageries of Sentinel-2A $(10 \mathrm{~m})$, pixel-based classification methods are more suitable than OBIA. SVM is still the best performing classifier, especially in pixel-based classification. The improved spatial resolution of Sentinel-2A enhances visual interpretation and classification. However, the image fusion of Landsat-8 and Sentinel-2A has been unable to provide a better quality result for single-scene/single-temporal application. Nevertheless, it is useful for multitemporal analysis involving the use of archived Landsat and Sentinel data.

The use of Sentinel-2A images to produce more accurate mapping of urban land should be explored in the future. Future works can also extend the analysis over a large number of urban areas using various algorithms to test the Sentinel-2A performance comprehensively. Other application areas can also be tested and analysed to fully evaluate the mapping capability of Sentinel-2A in Malaysia.

\section{Acknowledgements}

Sentinel-2A was downloaded from Copernicus Open Access Hub from ESA, and Landsat-8 data was obtained through USGS, Earth Resources Observation and Science Centre. The authors also acknowledge the facilities and financial support provided by Universiti Putra Malaysia (UPM) and Malaysian Space Agency (MYSA).

\section{References}

[1] F Pacifici, M Chini and WJ Emery. A neural network approach using multi-scale textural metrics from very high-resolution panchromatic imagery for urban land-use classification. Rem. Sens. Environ. 2009; 113, 1276-92.

[2] HZM Shafri, E Taherzadeh, S Mansor and R Ashurov. Hyperspectral remote sensing of urban areas: An overview of techniques and applications. Res. J. Appl. Sci. Eng. Tech. 2012; 4, 1557-65.

[3] K Jia, X Wei, X Gu, Y Yao, X Xie and B Li. Land cover classification using Landsat 8 Operational Land Imager data in Beijing, China. Geocarto Int. 2014; 29, 941-51.

[4] MN Tuanmu and W Jetz. A global 1-km consensus land-cover product for biodiversity and ecosystem modelling. Glob. Ecol. Biogeogr. 2014; 23, 1031-45.

[5] S Gadal and W Ouerghemmi. Morpho-spectral recognition of dense urban objects by hyperspectral imagery. Int. Arch. Photogram. Rem. Sens. Spatial Inf. Sci. 2015; XL-3/W3, 433-8

[6] G Suresh, R Gehrke, T Wiatr and M Hovenbitzer. Synthetic aperture radar (SAR) based classifiers for land applications in Germany. Int. Arch. Photogram. Rem. Sens. Spatial Inf. Sci. 2016; XLI-B1, 1187-93.

[7] XY Wang, YG Guo, J He and LT Du. Fusion of HJ1B and ALOS PALSAR data for land cover classification using machine learning methods. Int. J. Appl. Earth Observat. Geoinform. 2016; 52, 192-203.

[8] M Pesaresi, C Corbane, A Julea, AJ Florczyk, V Syrris and P Soille. Assessment of the added-value of Sentinel-2 for detecting built-up areas. Rem. Sens. 2016; 8, 299.

[9] J Haas and Y Ban. Sentinel-1A SAR and Sentinel-2A MSI data fusion for urban ecosystem service mapping. Rem. Sens. Appl. Soc. Environ. 2017; 8, 41-53.

[10] J Radoux, G Chomé, DC Jacques, F Waldner, N Bellemans, N Matton, C Lamarche, R D'Andrimont and P Defourny. Sentinel-2's potential for sub-pixel landscape feature detection. Rem. Sens. 2016; 8, 488.

[11] N Clerici, CAV Calderón and JM Posada. Fusion of sentinel-1A and sentinel-2A data for land cover mapping: A case study in the lower Magdalena region, Colombia. J. Map. 2017; 13, 718-26.

[12] DC Duro, SE Franklin and MG Dubé. A comparison of pixel-based and object-based image analysis with selected machine learning algorithms for the classification of agricultural landscapes using SPOT-5 HRG imagery. Rem. Sens. Environ. 2012; 118, 259-72.

[13] SW Myint, P Gober, A Brazel, S Grossman-Clarke and Q Weng. Per-pixel vs. object-based classification of urban land cover extraction using high spatial resolution imagery. Rem. Sens. Environ. 2011; 115, 1145-61.

[14] MBA Gibril, SA Bakar, K Yao, MO Idrees and B Pradhan. Fusion of RADARSAT-2 and multispectral optical remote sensing data for LULC extraction in a tropical agricultural area. Geocarto Int. 2017; 32, 735-48.

[15] F Pirotti, F Sunar and M Piragnolo. Benchmark of machine learning methods for classification of a Sentinel-2 image. Int. Arch. Photogram. Rem. Sens. Spatial Inf. Sci. 2016; XLI-B7, 335-40

[16] RH Topaloğlu, E Sertel and N Musaoğlu. Assessment of classification accuracies of Sentinel-2 and Landsat-8 data for land cover/use mapping. Int. Arch. Photogram. Rem. Sens. Spatial Inf. Sci. 2016; 
XLI-B8, 1055-9.

[17] D Liu and F Xia. Assessing object-based classification: Advantages and limitations. Rem. Sens. Lett. 2010; 1, 187-94.

[18] Q Wang, GA Blackburn, AO Onojeghuo, J Dash, L Zhou, Y Zhang and PM Atkinson. Fusion of Landsat 8 OLI and Sentinel-2 MSI data. IEEE Trans. Geosci. Rem. Sens. 2017; 5, 3885-99.

[19] M Drusch, UD Bello, S Carlier, O Colin, V Fernandez, F Gascon, B Hoersch, C Isola, P Laberinti, P Martimort, A Meygret, F Spoto, O Sy, F Marchese and P Bargellini. Sentinel-2: ESA's optical high-resolution mission for GMES operational services. Rem. Sens. Environ. 2012; 120, 25-36.

[20] O Hagolle, S Sylvander, M Huc, M Claverie, D Clesse, C Dechoz, V Lonjou and V Poulain. SPOT4 (take 5): Simulation of sentinel-2 time series on 45 large sites. Rem. Sens. 2015; 7, 12242-64.

[21] AD Vaiopoulos and K Karantzalos. Pansharpening on the narrow VNIR and SWIR spectral bands of Sentinel-2. Int. Arch. Photogram. Rem. Sens. Spatial Inf. Sci. 2016; XLI-B7, 723-30.

[22] CK Singh. Geospatial applications for natural resources management. CRC Press, Florida, 2018, p. $1-9$.

[23] J Zhang, C Pohl and JV Genderen. Remote sensing image fusion: A practical guide. CRC Press, Florida, 2017, p. 92-3.

[24] T Kuchma. Combined use of SAR and optical satellite images for landscape diversity assessment. In: Proceedings of the Living Planet Symposium, Prague, Czech Republic. 2016.

[25] A Abdulle, AA Tan, B Pradhan and S Abdullahi. Temporal assessment on land use land cover of Somalia after the effect of the civil war using remote sensing. In: Proceedings of the $8^{\text {th }}$ IGRSM International Conference and Exhibition on Geospatial \& Remote Sensing, Kuala Lumpur, Malaysia. 2016.

[26] S Moustakidis, G Mallinis, N Koutsias, JB Theocharis and V Petridis. SVM-based fuzzy decision trees for classification of high spatial resolution remote sensing images. IEEE Trans. Geosci. Rem. Sens. 2012; 50, 149-69.

[27] J Al-doski, SB Mansor and HZM Shafri. Image classification in remote sensing. J. Environ. Earth Sci. 2013; 3, 141-7.

[28] JR Jensen. Introductory digital image processing: A remote sensing perspective. $3^{\text {rd }}$ ed. Pearson Prentice Hall, New Jersey, 2015, p. 505-12.

[29] W Min. A Multiresolution Remotely Sensed Image Segmentation Method Combining Rainfalling Watershed Algorithm and Fast Region Merging. Int. Arch. Photogram. Rem. Sens. Spatial Inf. Sci. 2008; XXXVII, 1213-8. 